\title{
Yeni 2-Metoksi-6-[(3-alkil/aril-4,5-dihidro-1H-1,2,4-triazol-5-on-4-il)- azometin]fenil Furan-2-karboksilat Türevlerinin Sentezi ve in vitro Antioksidan Aktiviteleri
}

Abdurrahman Gürbüz ${ }^{1}$, Muzaffer Alkan², Sevda Manap ${ }^{3}$, Özlem Gürsoy Kol ${ }^{3 *}$, Gül Özdemir ${ }^{3}$, Haydar Yüksek ${ }^{3}$

$\quad \begin{aligned} & 1 \text { Kafkas University, Atatürk Health Services VC, } 36100 \text { Kars, Turkey (ORCID: 0000-0002-6820-6377) } \\ & \text { 2Kafkas University, Faculty of Education, 36100 Kars, Turkey (ORCID: 0000-0002-8820-4555) }\end{aligned}$
${ }^{3}$ Kafkas University, Department of Chemistry, 36100Kars, Turkey (ORCID: 0000-0002-5025-9622; 0000-0003-2637-9023; 0000-0001-7823-0920; 0000-00031289-1800)

(İlk Geliş Tarihi 16 Aralık 2019 ve Kabul Tarihi 13 Nisan 2020)

(DOI: 10.31590/ejosat.656126)

ATIF/REFERENCE: Gürbüz, A., Alkan, M., Manap, S., Kol, G. Ö., Özdemir, G. \& Yüksek, H. (2019). Synthesis and in vitro Antioxidant Activities of Novel 2-Methoxy-6-[(3-alkyl/aryl-4,5-dihydro-1H-1,2,4-triazol-5-on-4-yl)-azomethine]phenyl Furan-2carboxylate Derivatives. Avrupa Bilim ve Teknoloji Dergisi, (18), 1004-1011.

Öz

1,2,4-Triazol ve 4,5-dihidro-1H-1,2,4-triazol-5-on halkası taşıyan heterosiklik bileşikler çok geniş bir spektrumda biyolojik aktivite göstermektedir. Schiff bazlarının ise boya, tekstil, kozmetik, analitik reaktifler, su iyileştirme ürünleri, petrol katkı maddeleri, sentetik polimer ürünleri, deri ve kağıt gibi doğal makromoleküler materyallerin iyileştirilmesi gibi çeşitli pratik uygulamaları bulunmaktadır. Ancak Schiff Bazlarının en önemli uygulama alanı ilaç kimyasıdır. Potansiyel biyolojik aktif 1,2,4-triazol halkası içeren Schiff bazlarının birçok hastalığın tedavisinde kullanılabilecek faydalı moleküllerin ve ileride hastalıkların tedavisinde kullanılabilecek ilaçların elde edilmesi ümit edilmektedir. Bu amaçla 3-alkil(aril)-4-amino-4,5-dihidro-1H-1,2,4-triazol-5-on'ların (1), 2-formil-6metoksifenil furan-2-karboksilat (2) ile reaksiyonundan 2-metoksi-6-[(3-alkil/aril-4,5-dihidro-1H-1,2,4-triazol-5-on-4-il)azometin]fenil furan-2-karboksilatlar (3) elde edilmiştir. Daha sonra 3 bileşiklerinin asetilasyon reaksiyonları incelenerek 2-metoksi-6[(1-asetil-3-alkil/aril-4,5-dihidro-1H-1,2,4-triazol-5-on-4-il)-azometin]fenil furan-2-karboksilatlar (4) sentezlenmiştir. On dört yeni bileşiğin yapıları IR, ${ }^{1} \mathrm{H}$ NMR, ${ }^{13} \mathrm{C}$ NMR ve UV spektral verileri kullanılarak aydınlatılmıştır. Ayrıca, sentezlenen 3 ve 4 tipi yeni bileşiklerin üç farklı yöntemle (indirgeme gücü, serbest radikal giderme aktivitesi ve metal şelat aktivitesi) in vitro antioksidan özellikleri değerlendirilmiştir.

Anahtar Kelimeler: Schiff baz1, 4,5-Dihidro-1H-1,2,4-triazol-5-on, Sentez, Asetilasyon, Antioksidan Aktivitesi.

\section{Synthesis and in vitro Antioxidant Activities of Novel 2-Methoxy-6-[(3- alkyl/aryl-4,5-dihydro-1 $H$-1,2,4-triazol-5-on-4-yl)-azomethine]phenyl Furan-2-carboxylate Derivatives}

\begin{abstract}
Heterocyclic compounds bearing 1,2,4-triazole and 4,5-dihydro- $1 H$-1,2,4-triazole-5-one ring show a wide spectrum of biological activity. Schiff bases have various practical applications such as dyes, textile, cosmetics, analytical reagents, water treatment products, petroleum additives, synthetic polymer products, the improvement of natural macromolecular materials like leather and paper. However, the most important application area of Schiff Bases is pharmaceutical chemistry. It is hoped that Schiff bases containing a potentially biologically active 1,2,4-triazole ring will provide useful molecules that can be used in the treatment of many diseases and drugs that can be used in the treatment of future diseases. In this regard, new 2-methoxy-6-[(3-alkyl/aryl-4,5-dihydro-1H-1,2,4-triazol-5-on-4-yl)azomethine]phenyl furan-2-carboxylates (3) were obtained from the reactions of 3-alkyl(aryl)-4-amino-4,5-dihydro-1 H-1,2,4-triazol-5ones (1) with 2-formyl-6-methoxyphenyl furan-2-carboxylate (2). Then, acetylation reactions of $\mathbf{3}$ compounds were examined and 2methoxy-6-[(1-acetyl-3-alkyl/aryl-4,5-dihydro-1H-1,2,4-triazol-5-on-4-yl)-azomethine]phenyl furan-2-carboxylates (4) were
\end{abstract}

* Sorumlu Yazar: Kafkas University, Department of Chemistry, 36100Kars, Turkey, ORCID: 0000-0003-2637-9023, ozlemgursoy@gmail.com 
synthesized. The structures of fourteen new compounds were characterized by IR, ${ }^{1} \mathrm{H}$ NMR, ${ }^{13} \mathrm{C}$ NMR and UV spectral data. Besides, in vitro antioxidant properties of the newly synthesized $\mathbf{3}$ and $\mathbf{4}$ type compounds were evaluated by three different methods (reduction power, free radical removal activity and metal chelate activity).

Keywords: Schiff base, 4,5-Dihydro-1H-1,2,4-triazol-5-one, Syntheses, Acetylation, Antioxidant Activity.

\section{Giriş}

Serbest radikaller, biyolojik membranlarda lipid peroksidasyonuna, protein oksidasyonuna ve DNA hasarına sebep olarak hücre metabolizmasını olumsuz yönde etkilerler. Fizyolojik koşullarda, oksidan etkenler ve antioksidan mekanizmalar bir denge halinde bulunmaktadır. Fizyopatolojik şartlarda biyomoloküllerin oksidasyonunu geciktiren veya engelleyen endojen ve eksojen antioksidanlara son yıllarda ilgi giderek artmakta ve organizmaya zarar vermeyen antioksidan özellikteki sentetik bileşiklerin üretimi ve biyolojik sistemlerde oksidan-antioksidan denge üzerine olan etkileri araştırılmaktadır. Eksojen ve metabolik reaksiyonlar sonucu oluşan endojen kimyasallar oldukça reaktif olan serbest radikalleri oluşturabilmektedirler. Özellikle oksijen orijinli radikaller hücre hasarı ve hücre ölümüne sebep olabilecek kadar biyomolekülleri okside edebilme özelliklerine sahiptirler. Oksidatif stres, hastalıkların patolojisinde önemli rol oynar. Reaktif oksijen (ROS) ve azot türlerinin (RNS) aşırı üretimi ve aynı zamanda antioksidan kapasitenin yetersiz olması organizmalarda enflamasyon, diabet, genotoksisite ve kanser gibi çeşitli fizyopatolojik olaylara sebep olur (Hussain ve ark., 2003; McClements ve Decker, 2000).

1,2,4-Triazol türevlerinin antibakteriyel, antioksidan, antienflamatuar, antiparaziter, analjezik, antiviral, antitümör, anti-HIV, antihipertansif ve diüretik özellikleri gibi çok çeşitli farmakolojik aktivitelere sahip oldukları bilinmektedir (Aktaş-Yokuş ve ark., 2017; Ali ve ark., 2011; Chidananda ve ark., 2012; Demirbaş, Uğurluoğlu ve Demirbaş, 2002; Gürsoy-Kol ve Ayazoğlu 2017; Henen ve ark., 2012; İkizler ve ark., 1999; Li ve ark., 2013; Saadeh ve ark., 2010; Uzgören-Baran ve ark., 2012; Yüksek ve ark, 2013). Ayrıca, yine 1,2,4-triazol halkasını içeren $\mathrm{N}$-arilidenamino-4,5-dihidro- $1 \mathrm{H}$-1,2,4-triazol-5-on türevlerinin sentezini bildiren bazı makaleler de mevcuttur (Bahçeci ve ark., 2002; Gürsoy-Kol ve Ayazoğlu, 2017; Yüksek ve ark., 2005). 4,5-Dihidro-1H-1,2,4-triazol-5-on türevlerinin asetilasyonu ile ilgili çalışmalar da bildirilmiştir (Alkan ve ark., 2008; İkizler ve ark., 1993; Gürsoy-Kol ve Yüksek, 2010).

Aldehid ve ketonların primer aminler ile reaksiyonlarından oluşan imin tipi Schiff bazları kimyanın birçok alanında, tıpta, sanayide, teknolojide geniş bir kullanım alanı bulmuştur. Bu çalışmada, trietilamin kullanılarak 2-furoil klorür ile 2-hidroksi-3metoksibenzaldehidin reaksiyonundan 2-formil-6-metoksifenil furan-2-karboksilat (2) sentezlenmiştir. Bir primer amin gibi hareket eden ve $\mathrm{N}-\mathrm{NH}_{2}$ grubu içeren 3-alkil(aril)-4-amino-4,5-dihidro-1H-1,2,4-triazol-5-on'ların (1), 2 tipi aldehid ile reaksiyonları incelenerek heteroaromatik Schiff bazları olan 2-metoksi-6-[(3-alkil/aril-4,5-dihidro-1H-1,2,4-triazol-5-on-4-il)-azometin]fenil furan2-karboksilatlar (3) elde edilmiştir. Ayrıca, 3 tipi bileşiklerin asetik anhidrit ile reaksiyonları incelenmiş ve 4 tipi bileşikler hazırlanmıştır (Şema 1). Başlangıç bileşikleri (1), literatüre uygun olarak (İkizler ve Ün 1979; İkizler ve Yüksek 1993) ilgili ester etoksikarbonilhidrazonların sulu hidrazin hidrat çözeltisi ile reaksiyonlarından hazırlanmıştır. Yeni bileşiklerin yapıları IR, ${ }^{1} \mathrm{H}$ NMR, ${ }^{13} \mathrm{C}$ NMR, UV spektral verileri kullanılarak aydınlatılmıştır. 3 ve 4 Tipi bileşiklerin antioksidan aktiviteleri üç farklı yöntem (indirgeme gücü, serbest radikal giderme aktivitesi ve metal şelatlama aktivitesi) kullanılarak analiz edilmiştir.

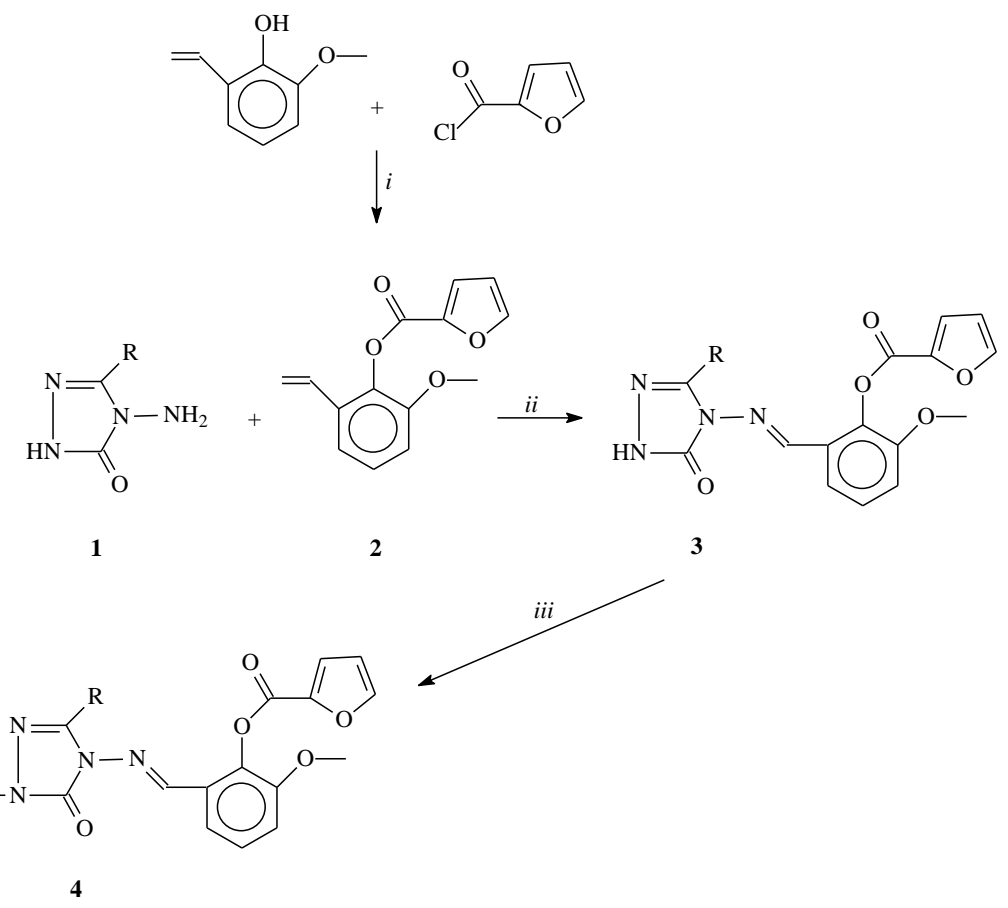

i) $\mathrm{Et}_{3} \mathrm{~N}$; ii) AcOH, reflaks, 1h; iii) $\mathrm{Ac}_{2} \mathrm{O}$, reflaks

a) $\mathrm{R}=\mathrm{CH}_{3}$, b) $\mathrm{R}=\mathrm{CH}_{2} \mathrm{CH}_{3}$, c) $\mathrm{R}=\mathrm{CH}_{2} \mathrm{CH}_{2} \mathrm{CH}_{3}$, d) $\left.\mathrm{R}=\mathrm{CH}_{2} \mathrm{C}_{6} \mathrm{H}_{5}, \mathrm{e}\right) \mathrm{R}=\mathrm{CH}_{2} \mathrm{C}_{6} \mathrm{H}_{4} \mathrm{CH}_{3}(p-)$,

f) $\left.\mathrm{R}=\mathrm{CH}_{2} \mathrm{C}_{6} \mathrm{H}_{4} \mathrm{Cl}(p-), \mathrm{g}\right) \mathrm{R}=\mathrm{C}_{6} \mathrm{H}_{5}$

Şema 1: 2-4 bileşiklerinin sentezi 


\section{Materyal ve Metot}

Bu çalışmada kullanılan kimyasal reaktifler Merck AG, Aldrich ve Fluka'dan satın alınmıştır. Erime noktaları, Stuart SMP30 erime noktası aparatı kullanılarak açık cam kapiler kullanılarak belirlenmiştir. İnfrared spektrum verileri Alpha-P Bruker FT-IR spektrometresi ile kaydedilmiştir. ${ }^{1} \mathrm{H}$ ve ${ }^{13} \mathrm{C}$ NMR spektrumları, dötero dimetil sülfoksit ( $\mathrm{d}_{6}$-DMSO) içerisinde iç standart olarak tetrametilsilan (TMS) kullanılarak, Bruker Avance III spektrofotometresi ile alınmıştır.

\subsection{2-Metoksi-6-[(3-alkil/aril-4,5-dihidro-1H-1,2,4-triazol-5-on-4-il)-azometin]fenil furan-2 karboksilatların (3) sentezi için genel yöntem}

Etil asetat $(50 \mathrm{~mL})$ içinde çözülmüş 2-hidroksi-3-metoksi-benzaldehid $(0.01 \mathrm{~mol})$, 2-furoil klorür $(0.01 \mathrm{~mol})$ ile muamele edilmiş ve bu çözeltiye, yavaş yavaş $0-5^{\circ} \mathrm{C}$ 'de karıştırılarak $10 \mathrm{~mL}$ etil asetat içerisinde trietilamin $(0.01 \mathrm{~mol})$ ilave edilmiştir. Karıştırma 2 saat devam etmiş ve sonra karışım 3 saat reflaks yapılarak süzülmüştür. Süzüntü vakum altında buharlaştırılmış, ham ürün suyla yıkanmış

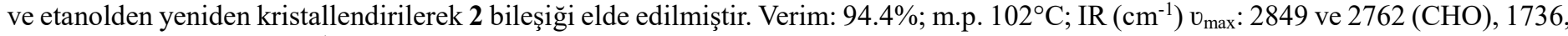
$1696(\mathrm{C}=\mathrm{O}), 1250(\mathrm{COO}) .{ }^{1} \mathrm{H}-\mathrm{NMR}\left(400 \mathrm{MHz}, \mathrm{DMSO}-\mathrm{d}_{6}\right)(\mathrm{ppm}) \delta \mathrm{H}: 3.87\left(\mathrm{~s}, 3 \mathrm{H}, \mathrm{OCH}_{3}\right), 6.85(\mathrm{dd}, 1 \mathrm{H}, \mathrm{ArH}, J=3.60,2.00 \mathrm{~Hz}), 7.52-$ $7.58(\mathrm{~m}, 3 \mathrm{H}, \mathrm{ArH}), 7.64(\mathrm{dd}, 1 \mathrm{H}, \mathrm{ArH}, J=3.60,0.80 \mathrm{~Hz}), 8.15(\mathrm{~m}, 1 \mathrm{H}, \mathrm{ArH}), 10.12(\mathrm{~s}, 1 \mathrm{H}, \mathrm{CHO}) ;{ }^{13} \mathrm{C}-\mathrm{NMR}\left(100 \mathrm{MHz}, \mathrm{DMSO}-\mathrm{d}_{6}\right)$ $(\mathrm{ppm}) \delta \mathrm{C}: 56.39\left(\mathrm{OCH}_{3}\right),[112.82,118.76,120.71,121.69,127.51,129.09,139.24,142.46,148.86,151.59]$ (ArC), 155.53 (COO), 189.85 (CHO). Daha sonra, 1 bileşiği $(0.01 \mathrm{~mol})$ asetik asit ile çözünmüş $(20 \mathrm{~mL})$ ve 2-formil-6-metoksifenil furan-2-karboksilat (2) (0.01 mol) ile muamele edilmiştir. Karışım 2 saat reflaks yapılmış ve $50-55^{\circ} \mathrm{C}$ 'de vakumda kurutulmuştur. Katı madde birkaç kez etanol ile kristallendirilerek, renksiz kristaller olan 3 tipi bileşikler elde edilmiştir.

\section{2-Metoksi-6-[(3-metil-4,5-dihidro-1H-1,2,4-triazol-5-on-4-il)-azometin]fenil furan-2-karboksilat (3a)}

Verim: 96.5\%, m.p. $226^{\circ} \mathrm{C}$. IR $\left(\mathrm{KBr}, v, \mathrm{~cm}^{-1}\right): 3148(\mathrm{NH}), 1746,1696(\mathrm{C}=\mathrm{O}), 1607(\mathrm{C}=\mathrm{N}), 1286(\mathrm{COO}) .{ }^{1} \mathrm{H}-\mathrm{NMR}(200 \mathrm{MHz}$, $\left.\mathrm{DMSO}_{6}\right)(\mathrm{ppm}) \delta \mathrm{H}: 2.07\left(\mathrm{~s}, 3 \mathrm{H}, \mathrm{CH}_{3}\right), 3.77\left(\mathrm{~s}, 3 \mathrm{H}, \mathrm{OCH}_{3}\right), 6.78(\mathrm{~m}, 1 \mathrm{H}, \mathrm{ArH}), 7.31-7.58(\mathrm{~m}, 4 \mathrm{H}, \mathrm{ArH}), 8.09(\mathrm{~m}, 1 \mathrm{H}, \mathrm{ArH}), 9.81(\mathrm{~s}$, $1 \mathrm{H}, \mathrm{N}=\mathrm{CH}), 11.77(\mathrm{~s}, 1 \mathrm{H}, \mathrm{NH})$; UV [Etanol, $\lambda_{\max }, \mathrm{nm}\left(\varepsilon, \mathrm{L}_{\mathrm{mol}}{ }^{-1} \cdot \mathrm{cm}^{-1}\right)$ ]: 298 (23.200), 258 (31.500), 214 (22.550).

\section{2-Metoksi-6-[(3-etil-4,5-dihidro-1H-1,2,4-triazol-5-on-4-il)-azometin]fenil furan-2-karboksilat (3b)}

Verim 92.7\%, m.p. $202^{\circ} \mathrm{C}$. IR (KBr, v, $\left.\mathrm{cm}^{-1}\right): 3185(\mathrm{NH}), 1742,1696(\mathrm{C}=\mathrm{O}), 1607,1589(\mathrm{C}=\mathrm{N}), 1278(\mathrm{COO}) .{ }^{1} \mathrm{H}-\mathrm{NMR}(200 \mathrm{MHz}$, DMSO-d 6 (ppm) $\delta \mathrm{H}: 1.02-1.09\left(\mathrm{~m}, 3 \mathrm{H}, \mathrm{CH}_{2} \mathrm{CH}_{3}\right), 2.38-2.45\left(\mathrm{~m}, 3 \mathrm{H}, \mathrm{CH}_{2} \mathrm{CH}_{3}\right), 3.76(\mathrm{~s}, 3 \mathrm{H}, \mathrm{OCH}), 6.77-6.78(\mathrm{~m}, 1 \mathrm{H}, \mathrm{ArH}), 7.28-$ $7.54(\mathrm{~m}, 4 \mathrm{H}, \mathrm{ArH}), 8.09-8.10(\mathrm{~m}, 1 \mathrm{H}, \mathrm{ArH}), 9.80(\mathrm{~s}, 1 \mathrm{H}, \mathrm{N}=\mathrm{CH}), 11.79(\mathrm{~s}, 1 \mathrm{H}, \mathrm{NH})$; UV [Etanol, $\left.\lambda_{\max }, \mathrm{nm}\left(\varepsilon, \mathrm{L}_{\mathrm{mol}}{ }^{-1} \cdot \mathrm{cm}^{-1}\right)\right]: 296$ (20.410), 258 (30.260), 216 (22.530).

\section{2-Metoksi-6-[(3-n-propil-4,5-dihidro-1H-1,2,4-triazol-5-on-4-il)-azometin]fenil furan-2-karboksilat (3c)}

Verim 89.2\%, m.p. $204^{\circ} \mathrm{C}$. IR (KBr, v, $\left.\mathrm{cm}^{-1}\right): 3164(\mathrm{NH}), 1751,1698(\mathrm{C}=\mathrm{O}), 1606,1589(\mathrm{C}=\mathrm{N}), 1282(\mathrm{COO}) .{ }^{1} \mathrm{H}-\mathrm{NMR}(200 \mathrm{MHz}$, DMSO-d $)$ (ppm) $\delta \mathrm{H}: 0.83$ (t, 3H, $\mathrm{CH}_{2} \mathrm{CH}_{2} \mathrm{CH}_{3}, J=7.40 \mathrm{~Hz}$ ), 1.57 (sext, $\left.3 \mathrm{H}, \mathrm{CH}_{2} \mathrm{CH}_{2} \mathrm{CH}_{3}, J=7.40 \mathrm{~Hz}\right), 2.40\left(\mathrm{q}, 2 \mathrm{H}, \mathrm{CH}_{2} \mathrm{CH}_{2} \mathrm{CH}_{3}, J\right.$ $=7.40 \mathrm{~Hz}), 3.75\left(\mathrm{~s}, 3 \mathrm{H}, \mathrm{OCH}_{3}\right), 6.76-6.80(\mathrm{~m}, 1 \mathrm{H}, \mathrm{ArH}), 7.27-7.59(\mathrm{~m}, 4 \mathrm{H}, \mathrm{ArH}), 8.10(\mathrm{~m}, 1 \mathrm{H}, \mathrm{ArH}), 9.79(\mathrm{~s}, 1 \mathrm{H}, \mathrm{N}=\mathrm{CH}), 11.80(\mathrm{~s}$, $1 \mathrm{H}, \mathrm{NH})$; UV [Etanol, $\left.\lambda_{\max }, \mathrm{nm}\left(\varepsilon, \mathrm{L} \cdot \mathrm{mol}^{-1} . \mathrm{cm}^{-1}\right)\right]: 298$ (13.070), 256 (26.630), 226 (50.510), 216 (18.970).

\section{2-Metoksi-6-[(3-benzil-4,5-dihidro-1H-1,2,4-triazol-5-on-4-il)-azometin]fenil furan-2-karboksilat (3d)}

Verim 98.1\%, m.p. $209^{\circ} \mathrm{C}$. IR (KBr, $\left.v, \mathrm{~cm}^{-1}\right): 3136(\mathrm{NH}), 1743,1715(\mathrm{C}=\mathrm{O}), 1588(\mathrm{C}=\mathrm{N}), 1280(\mathrm{COO}), 769$ ve 705 (monosubstitue benzenoid halka). ${ }^{1} \mathrm{H}-\mathrm{NMR}\left(200 \mathrm{MHz}, \mathrm{DMSO}-\mathrm{d}_{6}\right)(\mathrm{ppm}) \delta \mathrm{H}: 3.75\left(\mathrm{~s}, 3 \mathrm{H}, \mathrm{OCH}_{3}\right), 3.89\left(\mathrm{~s}, 2 \mathrm{H}, \mathrm{CH}_{2} \mathrm{Ph}\right), 6.75-6.78(\mathrm{~m}, 1 \mathrm{H}, \mathrm{ArH}), 7.16-$ $7.58(\mathrm{~m}, 9 \mathrm{H}, \mathrm{ArH}), 8.07-8.08(\mathrm{~m}, 1 \mathrm{H}, \mathrm{ArH}), 9.78(\mathrm{~s}, 1 \mathrm{H}, \mathrm{N}=\mathrm{CH}), 11.91(\mathrm{~s}, 1 \mathrm{H}, \mathrm{NH})$; UV [Etanol, $\left.\lambda_{\max }, \mathrm{nm}\left(\varepsilon, \mathrm{L}^{\mathrm{mol}}{ }^{-1} . \mathrm{cm}^{-1}\right)\right]: 298$ (17.000), 254 (30.070), 230 (25.220), 214 (22.480).

\section{2-Metoksi-6-[(3-p-metilbenzil-4,5-dihidro-1H-1,2,4-triazol-5-on-4-il)-azometin]-fenil furan-2-karboksilat (3e)}

Verim 98.4\%, m.p. $202^{\circ} \mathrm{C}$. IR (KBr, v, cm $\left.{ }^{-1}\right): 3165(\mathrm{NH}), 1757,1704(\mathrm{C}=\mathrm{O}), 1591(\mathrm{C}=\mathrm{N}), 1283$ (COO), $825(1,4-$ disubstitue benzenoid halka). ${ }^{1} \mathrm{H}-\mathrm{NMR}\left(200 \mathrm{MHz}, \mathrm{DMSO}-\mathrm{d}_{6}\right)(\mathrm{ppm}) \delta \mathrm{H}: 2.20\left(\mathrm{~s}, 3 \mathrm{H}, \mathrm{PhCH}_{3}\right), 3.76\left(\mathrm{~s}, 3 \mathrm{H}, \mathrm{OCH}_{3}\right), 3.84\left(\mathrm{~s}, 2 \mathrm{H}, \mathrm{CH}_{2} \mathrm{Ph}\right), 6.76(\mathrm{~m}$, $1 \mathrm{H}, \mathrm{ArH}), 7.05-7.10$ (m, 4H, ArH), 7.31-7.59 (m, 4H, ArH), 8.05-8.09 (m, 1H, ArH), 9.81 (s, 1H, N=CH), 11.89 (s, 1H, NH); UV [Etanol, $\lambda_{\max }, \mathrm{nm}\left(\varepsilon, \mathrm{L}_{\mathrm{mol}}{ }^{-1} \cdot \mathrm{cm}^{-1}\right)$ ]: 298 (17.030), 256 (30.290), 230 (25.430), 216 (23.210).

\section{2-Metoksi-6-[(3-p-klorobenzil-4,5-dihidro-1H-1,2,4-triazol-5-on-4-il)-azometin]-fenyl furan-2-karboksilat (3f)}

Verim 97.3\%, m.p. $198^{\circ} \mathrm{C}$. IR ( KBr, v, $\left.\mathrm{cm}^{-1}\right): 3133(\mathrm{NH}), 1758,1704(\mathrm{C}=\mathrm{O}), 1592(\mathrm{C}=\mathrm{N}), 1284$ (COO), $822(1,4-$ disubstitue benzenoid halka). ${ }^{1} \mathrm{H}-\mathrm{NMR}\left(200 \mathrm{MHz}, \mathrm{DMSO}-\mathrm{d}_{6}\right)(\mathrm{ppm}) \delta \mathrm{H}: 3.76\left(\mathrm{~s}, 3 \mathrm{H}, \mathrm{OCH}_{3}\right), 3.90\left(\mathrm{~s}, 2 \mathrm{H}, \mathrm{CH}_{2} \mathrm{Ph}\right), 6.76-6.78(\mathrm{~m}, 1 \mathrm{H}, \mathrm{ArH}), 7.23-$ $7.58(\mathrm{~m}, 8 \mathrm{H}, \mathrm{ArH}), 8.08(\mathrm{~m}, 1 \mathrm{H}, \mathrm{ArH}), 9.78(\mathrm{~s}, 1 \mathrm{H}, \mathrm{N}=\mathrm{CH}), 11.94(\mathrm{~s}, 1 \mathrm{H}, \mathrm{NH}) ; \mathrm{UV}$ [Etanol, $\left.\lambda_{\max }, \mathrm{nm}\left(\varepsilon, \mathrm{L}_{\mathrm{mol}}{ }^{-1} . \mathrm{cm}^{-1}\right)\right]: 298(13.760)$, 258 (27.090), $226(23.970), 214(21.280)$.

\section{2-Metoksi-6-[(3-fenil-4,5-dihidro-1 H-1,2,4-triazol-5-on-4-il)-azometin]fenil furan-2-karboksilat (3g)}

Verim 97.8\%, m.p. $218^{\circ} \mathrm{C}$. IR (KBr, $\left.v, \mathrm{~cm}^{-1}\right): 3213(\mathrm{NH}), 1742,1708(\mathrm{C}=\mathrm{O}), 1612(\mathrm{C}=\mathrm{N}), 1278(\mathrm{COO}), 768$ ve 696 (monosubstitue benzenoid halka). ${ }^{1} \mathrm{H}-\mathrm{NMR}\left(200 \mathrm{MHz}, \mathrm{DMSO}-\mathrm{d}_{6}\right)(\mathrm{ppm}) \delta \mathrm{H}: 3.78\left(\mathrm{~s}, 3 \mathrm{H}, \mathrm{OCH}_{3}\right), 6.75-6.77(\mathrm{~m}, 1 \mathrm{H}, \mathrm{ArH}), 7.28-7.55(\mathrm{~m}, 7 \mathrm{H}, \mathrm{ArH})$, 7.78-7.83 (m, 2H, ArH), $8.08(\mathrm{~m}, 1 \mathrm{H}, \mathrm{ArH}), 9.78(\mathrm{~s}, 1 \mathrm{H}, \mathrm{N}=\mathrm{CH}), 12.31(\mathrm{~s}, 1 \mathrm{H}, \mathrm{NH}) ; \mathrm{UV}$ [Etanol, $\left.\lambda_{\max }, \mathrm{nm}\left(\varepsilon, \mathrm{L}_{\mathrm{mol}}{ }^{-1} . \mathrm{cm}^{-1}\right)\right]: 282$ (28.990), $256(30.210), 216(22.900)$. 


\subsection{2-Metoksi-6-[(1-asetil-3-alkil/aril-4,5-dihidro-1H-1,2,4-triazol-5-on-4-il)-azometin]fenil furan-2- karboksilatların (4) sentezi için genel yöntem}

3 Bileşiği $(0.01 \mathrm{~mol})$, otuz dakika boyunca asetik anhidrit $(15 \mathrm{~mL})$ ile reflaks yapılmıştır. Daha sonra mutlak etanolün $(50 \mathrm{~mL})$ eklenmesiyle, karışım 1 saat daha reflaks yapılmıştır. Elde edilen çözeltinin $40-45^{\circ} \mathrm{C}$ 'de vakumda buharlaştırılması ve katının uygun bir çözücüden birkaç kere tekrar kristallendirilmesi ile saflaştırılmış olarak 4 bileşiği elde edilmiştir.

\section{2-Metoksi-6-[(1-asetil-3-metil-4,5-dihidro-1H-1,2,4-triazol-5-on-4-il)-azometin]-fenil furan-2-karboksilat (4a)}

Verim: 71.4\%, m.p. $182^{\circ} \mathrm{C}$. IR $\left(\mathrm{KBr}, v, \mathrm{~cm}^{-1}\right): 1728(\mathrm{C}=\mathrm{O}), 1625(\mathrm{C}=\mathrm{N}), 1295(\mathrm{COO}) .{ }^{1} \mathrm{H}-\mathrm{NMR}\left(200 \mathrm{MHz}, \mathrm{DMSO}-\mathrm{d}_{6}\right)(\mathrm{ppm}) \delta \mathrm{H}$ : $2.20\left(\mathrm{~s}, 3 \mathrm{H}, \mathrm{CH}_{3}\right), 2.43\left(\mathrm{~s}, 3 \mathrm{H}, \mathrm{COCH}_{3}\right), 3.83\left(\mathrm{~s}, 3 \mathrm{H}, \mathrm{OCH}_{3}\right), 6.84-6.87$ (m, 1H, ArH), 7.37-7.67 (m, 4H, ArH), 8.16-8.18 (m, 1H, ArH), $9.72(\mathrm{~s}, 1 \mathrm{H}, \mathrm{N}=\mathrm{CH})$.

\section{2-Metoksi-6-[(1-asetil-3-etil-4,5-dihidro-1H-1,2,4-triazol-5-on-4-il)-azometin]-fenil furan-2-karboksilat (4b)}

Verim 91.0\%, m.p. $168^{\circ} \mathrm{C}$. IR $\left(\mathrm{KBr}, v, \mathrm{~cm}^{-1}\right): 1747,1694(\mathrm{C}=\mathrm{O}), 1610(\mathrm{C}=\mathrm{N}), 1283(\mathrm{COO}) .{ }^{1} \mathrm{H}-\mathrm{NMR}\left(400 \mathrm{MHz}, \mathrm{DMSO}-\mathrm{d}_{6}\right)(\mathrm{ppm})$ $\delta \mathrm{H}: 1.17\left(\mathrm{t}, 3 \mathrm{H}, \mathrm{CH}_{2} \mathrm{CH}_{3}, J=7.20 \mathrm{~Hz}\right), 2.45\left(\mathrm{~s}, 3 \mathrm{H}, \mathrm{COCH}_{3}\right), 2.56\left(\mathrm{q}, 2 \mathrm{H}, \mathrm{CH}_{2} \mathrm{CH}_{3}, J=7.20 \mathrm{~Hz}\right), 3.83(\mathrm{~s}, 3 \mathrm{H}, \mathrm{OCH}), 6.86(\mathrm{dd}, 1 \mathrm{H}$, $\operatorname{ArH}, J=3.60,1.60 \mathrm{~Hz}), 7.35-7.41(\mathrm{~m}, 1 \mathrm{H}, \mathrm{ArH}), 7.45(\mathrm{t}, 1 \mathrm{H}, \mathrm{ArH}, J=8.00 \mathrm{~Hz}), 7.57(\mathrm{dd}, 1 \mathrm{H}, \mathrm{ArH}, J=8.00,1.60 \mathrm{~Hz}), 7.66(\mathrm{dd}, 1 \mathrm{H}$, $\mathrm{ArH}, J=3.60,0.80 \mathrm{~Hz}), 8.17(\mathrm{dd}, 1 \mathrm{H}, \mathrm{ArH}, J=2.00,0.80 \mathrm{~Hz}), 9.72(\mathrm{~s}, 1 \mathrm{H}, \mathrm{N}=\mathrm{CH}) ;{ }^{13} \mathrm{C}-\mathrm{NMR}\left(100 \mathrm{MHz}, \mathrm{DMSO}-\mathrm{d}_{6}\right)(\mathrm{ppm}) \delta \mathrm{C}: 9.79$ $\left(\mathrm{CH}_{2} \mathrm{CH}_{3}\right), 18.35\left(\mathrm{CH}_{2} \mathrm{CH}_{3}\right), 23.39\left(\mathrm{COCH}_{3}\right), 56.24\left(\mathrm{OCH}_{3}\right),[112.93,115.86,118.74,120.77,126.74,127.47,138.14,142.37,149.09$, 151.29] (ArC), 148.01 (triazol- $\left.\mathrm{C}_{3}\right), 150.18(\mathrm{~N}=\mathrm{CH}), 151.55$ (triazol-C5), $155.45(\mathrm{COO}), 165.98\left(\underline{\mathrm{COCH}}_{3}\right) ; \mathrm{UV}\left[\mathrm{Etanol}, \lambda_{\max }, \mathrm{nm}(\varepsilon\right.$, L. $\left.\left.\mathrm{mol}^{-1} . \mathrm{cm}^{-1}\right)\right]: 292(11.130), 256(19.170), 216(14.310)$.

\section{2-Metoksi-6-[(1-asetil-3-n-propil-4,5-dihidro-1H-1,2,4-triazol-5-on-4-il)-azometin]-fenil furan-2-karboksilat (4c)}

Verim 86.6\%, m.p. $140^{\circ} \mathrm{C}$. IR $\left(\mathrm{KBr}, v, \mathrm{~cm}^{-1}\right): 1734,1696(\mathrm{C}=\mathrm{O}), 1611(\mathrm{C}=\mathrm{N}), 1271(\mathrm{COO}) .{ }^{1} \mathrm{H}$ NMR $\left(400 \mathrm{MHz}, \mathrm{DMSO}-\mathrm{d}_{6}\right): \delta 0.95$ (t, $3 \mathrm{H}, \mathrm{CH}_{2} \mathrm{CH}_{2} \mathrm{CH}_{3} ; J=7.60 \mathrm{~Hz}$ ), 1.66 (sext, $\left.2 \mathrm{H}, \mathrm{CH}_{2} \underline{\mathrm{CH}}_{2} \mathrm{CH}_{3} ; J=7.20 \mathrm{~Hz}\right), 2.45\left(\mathrm{~s}, 3 \mathrm{H}, \mathrm{COCH}_{3}\right), 2.56\left(\mathrm{t}, 2 \mathrm{H}, \mathrm{CH}_{2} \mathrm{CH}_{2} \mathrm{CH}_{3} ; J=7.60 \mathrm{~Hz}\right)$, $3.84\left(\mathrm{~s}, 3 \mathrm{H}, \mathrm{OCH}_{3}\right), 6.86(\mathrm{dd}, 1 \mathrm{H}, \mathrm{ArH}, J=3.60,1.60 \mathrm{~Hz}), 7.39-7.41(\mathrm{~m}, 1 \mathrm{H}, \mathrm{ArH}), 7.46(\mathrm{t}, 1 \mathrm{H}, \mathrm{ArH}, J=8.00 \mathrm{~Hz}), 7.58(\mathrm{dd}, 1 \mathrm{H}, \mathrm{ArH}$, $J=8.00,1.60 \mathrm{~Hz}), 7.66(\mathrm{dd}, 1 \mathrm{H}, \mathrm{ArH}, J=3.60,0.80 \mathrm{~Hz}), 8.17(\mathrm{dd}, 1 \mathrm{H}, \mathrm{ArH}, J=1.60,0.80 \mathrm{~Hz}), 9.72(\mathrm{~s}, 1 \mathrm{H}, \mathrm{N}=\mathrm{CH}) ;{ }^{13} \mathrm{C} \mathrm{NMR}(100$ MHz, DMSO-d $\left.)_{6}\right): \delta 13.35\left(\mathrm{CH}_{2} \mathrm{CH}_{2} \underline{\mathrm{CH}_{3}}\right), 18.67\left(\mathrm{CH}_{2} \underline{\mathrm{CH}}_{2} \mathrm{CH}_{3}\right), 23.41\left(\mathrm{COCH}_{3}\right), 26.43\left(\underline{\mathrm{CH}}_{2} \mathrm{CH}_{2} \mathrm{CH}_{3}\right), 56.25\left(\mathrm{OCH}_{3}\right),[112.94,115.88$, $118.76,120.78,126.74,127.51,138.17,142.34,149.09,151.22](\mathrm{ArC}), 147.97\left(\right.$ triazol- $\left._{3}\right), 150.34(\mathrm{~N}=\mathrm{CH}), 151.55\left(\right.$ triazol- $\left.\mathrm{C}_{5}\right), 155.51$ (COO), $166.01\left(\underline{\mathrm{COCH}}_{3}\right)$; UV [Etanol, $\left.\lambda_{\max }, \mathrm{nm}\left(\varepsilon, \mathrm{L}_{\mathrm{mol}}{ }^{-1} . \mathrm{cm}^{-1}\right)\right]: 294$ (9.780), 254 (18.535), 218 (14.190).

\section{2-Metoksi-6-[(1-asetil-3-benzil-4,5-dihidro-1H-1,2,4-triazol-5-on-4-il)-azometin]-fenil furan-2-karboksilat (4d)}

Verim 81.8\%, m.p. $183^{\circ} \mathrm{C}$. IR $\left(\mathrm{KBr}, v, \mathrm{~cm}^{-1}\right)$ : 1739, $1715(\mathrm{C}=\mathrm{O}), 1609(\mathrm{C}=\mathrm{N}), 1251(\mathrm{COO}), 771$ ve 705 (monosubstitue benzenoid halka). ${ }^{1} \mathrm{H}$ NMR (400 MHz, DMSO-d $)$ ): $\delta 2.45\left(\mathrm{~s}, 3 \mathrm{H}, \mathrm{COCH}_{3}\right), 3.83\left(\mathrm{~s}, 3 \mathrm{H}, \mathrm{OCH}_{3}\right), 4.07\left(\mathrm{~s}, 6 \mathrm{H}, 3 \mathrm{CH}_{2}\right), 6.85(\mathrm{dd}, 1 \mathrm{H}, \mathrm{ArH}, J=3.60$, $1.60 \mathrm{~Hz}), 7.25-7.27$ (m, 1H, ArH), 7.28-7.33 (m, 4H, ArH), 7.36-7.40 (m, 1H, ArH), 7.45 (t, 1H, ArH, J=8.00 Hz), 7.55 (dd, 1H, ArH, $J=8.00,1.60 \mathrm{~Hz}), 7.66(\mathrm{dd}, 1 \mathrm{H}, \mathrm{ArH}, J=3.60,0.80 \mathrm{~Hz}), 8.16(\mathrm{dd}, 1 \mathrm{H}, \mathrm{ArH}, J=1.60,0.80 \mathrm{~Hz}), 9.72(\mathrm{~s}, 1 \mathrm{H}, \mathrm{N}=\mathrm{CH}) ;{ }^{13} \mathrm{C} \mathrm{NMR}(100$ MHz, DMSO-d $)_{6}: \delta 23.47\left(\mathrm{COCH}_{3}\right), 30.79\left(\mathrm{CH}_{2} \mathrm{Ph}\right), 56.26\left(\mathrm{OCH}_{3}\right),[112.97,115.91,117.89,121.03,126.76,127.49,138.47,142.09$, 148.06, 149.46] (arom-C), [126.96, $128.48(2 \mathrm{C}), 128.95(2 \mathrm{C}), 134.52]\left(\mathrm{C}_{3}\right.$ 'e bağlı arom-C), $147.97\left(\right.$ triazol- $\left.\mathrm{C}_{3}\right), 149.19(\mathrm{~N}=\mathrm{CH}), 151.46$ (triazol-C $\left.)_{5}\right), 155.38(\mathrm{COO}), 165.99\left(\mathrm{COCH}_{3}\right)$; UV [Etanol, $\left.\lambda_{\max }, \mathrm{nm}\left(\varepsilon, \mathrm{L}_{\mathrm{mol}}{ }^{-1} . \mathrm{cm}^{-1}\right)\right]: 292(9.110), 254(18.070), 226(15.725)$.

\section{2-Metoksi-6-[(1-asetil-3-p-metilbenzil-4,5-dihidro-1H-1,2,4-triazol-5-on-4-il)-azometin]-fenil furan-2-karboksilat (4e)}

Verim 78.8\%, m.p. $163^{\circ} \mathrm{C}$. IR (KBr, v, $\left.\mathrm{cm}^{-1}\right)$ : 1739, $1715(\mathrm{C}=\mathrm{O}), 1609(\mathrm{C}=\mathrm{N}), 1282(\mathrm{COO}), 819(1,4-$ disubstitue benzenoid halka). ${ }^{1} \mathrm{H}$ NMR $\left(400 \mathrm{MHz}, \mathrm{DMSO}-\mathrm{d}_{6}\right): \delta 2.28\left(\mathrm{~s}, 3 \mathrm{H}, \mathrm{PhCH}_{3}\right), 2.44\left(\mathrm{~s}, 3 \mathrm{H}, \mathrm{COCH}_{3}\right), 3.83\left(\mathrm{~s}, 3 \mathrm{H}, \mathrm{OCH}_{3}\right), 4.00\left(\mathrm{~s}, 2 \mathrm{H}, \mathrm{CH}_{2} \mathrm{Ph}\right), 6.84(\mathrm{dd}, 1 \mathrm{H}$, $\operatorname{ArH}, J=3.60,1.60 \mathrm{~Hz}), 7.14(\mathrm{~d}, 2 \mathrm{H}, \mathrm{ArH} ; J=7.60 \mathrm{~Hz}), 7.23(\mathrm{~d}, 2 \mathrm{H}, \mathrm{ArH} ; J=8.00 \mathrm{~Hz}), 7.38-7.40(\mathrm{~m}, 1 \mathrm{H}, \mathrm{ArH}), 7.46(\mathrm{t}, 1 \mathrm{H}, \mathrm{ArH}, J=$ $8.00 \mathrm{~Hz}), 7.56(\mathrm{dd}, 1 \mathrm{H}, \mathrm{ArH}, J=8.00,1.60 \mathrm{~Hz}), 7.65(\mathrm{dd}, 1 \mathrm{H}, \mathrm{ArH}, J=3.60,0.80 \mathrm{~Hz}), 8.16(\mathrm{dd}, 1 \mathrm{H}, \mathrm{ArH}, J=2.00,0.80 \mathrm{~Hz}), 9.71(\mathrm{~s}$, $1 \mathrm{H}, \mathrm{N}=\mathrm{CH}) ;{ }^{13} \mathrm{C}$ NMR $\left(100 \mathrm{MHz}, \mathrm{DMSO}-\mathrm{d}_{6}\right): \delta 20.62\left(\mathrm{PhCH}_{3}\right), 23.47\left(\mathrm{COCH}_{3}\right), 30.40\left(\mathrm{CH}_{2} \mathrm{Ph}\right), 56.26\left(\mathrm{OCH}_{3}\right),[112.96,115.90$, 117.94, 121.02, 126.76, 127.50, 138.46, 142.10, 148.21, 149.46] (arom-C), [128.83 (2C), 129.05 (2C), 131.37, 136.07] (C ${ }_{3}$ 'e bağlı arom-C), 147.97 (triazol-C 3$), 149.18(\mathrm{~N}=\mathrm{CH}), 151.47$ (triazol-C 5$), 155.38(\mathrm{COO}), 165.99\left(\mathrm{COCH}_{3}\right)$; UV [Etanol, $\lambda_{\max }, \mathrm{nm}\left(\varepsilon, \mathrm{L}_{\mathrm{mol}}{ }^{-}\right.$ $\left.{ }^{1} . \mathrm{cm}^{-1}\right)$ ]: 292 (13.930), 256 (27.490), 228 (25.030), $216(23.570)$.

\section{2-Metoksi-6-[(1-asetil-3-p-klorobenzil-4,5-dihidro-1H-1,2,4-triazol-5-on-4-il)-azometin]-fenil furan-2-karboksilat (4f)}

Verim 91.7\%, m.p. $178^{\circ} \mathrm{C}$. IR $\left(\mathrm{KBr}, v, \mathrm{~cm}^{-1}\right): 1731(\mathrm{C}=\mathrm{O}), 1607(\mathrm{C}=\mathrm{N}), 1290(\mathrm{COO}), 827\left(1,4\right.$-disubstitue benzenoid halka). ${ }^{1} \mathrm{H}$ NMR (400 MHz, DMSO-d $\left.)_{6}\right): \delta 2.44\left(\mathrm{~s}, 3 \mathrm{H}, \mathrm{COCH}_{3}\right), 3.83\left(\mathrm{~s}, 3 \mathrm{H}, \mathrm{OCH}_{3}\right), 4.07\left(\mathrm{~s}, 2 \mathrm{H}, \mathrm{CH}_{2} \mathrm{Ph}\right), 6.84(\mathrm{dd}, 1 \mathrm{H}, \mathrm{ArH}, J=3.60,1.60 \mathrm{~Hz})$, 7.36-7.42 (m, 5H, ArH), 7.45 (t, 1H, ArH, $J=8.00 \mathrm{~Hz}), 7.54$ (dd, 1H, ArH, $J=8.00,1.60 \mathrm{~Hz}), 7.66(\mathrm{dd}, 1 \mathrm{H}, \mathrm{ArH}, J=3.60,0.80 \mathrm{~Hz})$, $8.16(\mathrm{dd}, 1 \mathrm{H}, \mathrm{ArH}, J=1.60,0.80 \mathrm{~Hz}), 9.72(\mathrm{~s}, 1 \mathrm{H}, \mathrm{N}=\mathrm{CH}) ;{ }^{13} \mathrm{C}$ NMR $\left(100 \mathrm{MHz}, \mathrm{DMSO}-\mathrm{d}_{6}\right): \delta 23.46\left(\mathrm{COCH}_{3}\right), 30.11\left(\mathrm{CH}_{2} \mathrm{Ph}\right), 56.26$ $\left(\mathrm{OCH}_{3}\right),[112.97,115.94,117.95,121.03,126.72,127.51,138.46,142.09,147.96,149.52]$ (arom-C), [128.41 (2C), 130.90 (2C), 131.69, 133.52] ( $\mathrm{C}_{3}$ 'e bağl1 arom-C), 147.77 (triazol- $\left.\mathrm{C}_{3}\right), 149.19(\mathrm{~N}=\mathrm{CH}), 151.47$ (triazol-C 5$), 155.37(\mathrm{COO}), 165.96\left(\underline{\mathrm{COCH}}_{3}\right) ; \mathrm{UV}[\mathrm{Etanol}$, $\lambda_{\max }, \mathrm{nm}\left(\varepsilon,{\left.\left.\mathrm{L} . \mathrm{mol}^{-1} . \mathrm{cm}^{-1}\right)\right]:} 294\right.$ (11.030), 256 (22.600), 222 (26.240).

\section{2-Metoksi-6-[(1-asetil-3-fenil-4,5-dihidro-1H-1,2,4-triazol-5-on-4-il)-azometin]-fenil furan-2-karboksilat (4g)}

Verim $72.7 \%$, m.p. $204^{\circ} \mathrm{C}$. IR $\left(\mathrm{KBr}, v, \mathrm{~cm}^{-1}\right)$ : $1771,1736(\mathrm{C}=\mathrm{O}), 1607(\mathrm{C}=\mathrm{N}), 1194(\mathrm{COO}), 764$ ve 693 (monosubstitue benzenoid halka). ${ }^{1} \mathrm{H}$ NMR (400 MHz, DMSO-d $)$ ): $\delta 2.54\left(\mathrm{~s}, 3 \mathrm{H}, \mathrm{COCH}_{3}\right), 3.84\left(\mathrm{~s}, 3 \mathrm{H}, \mathrm{OCH}_{3}\right), 6.83(\mathrm{dd}, 1 \mathrm{H}, \mathrm{ArH}, J=3.60,1.60 \mathrm{~Hz}), 7.40-7.42$ $(\mathrm{m}, 1 \mathrm{H}, \mathrm{ArH}), 7.45$ (t, 1H, ArH, J=8.00 Hz), 7.51-7.61 (m, 5H, ArH), 7.90-7.92 (m, 2H, ArH), 8.15 (dd, 1H, ArH, J=2.00, 0.80 Hz), $9.69(\mathrm{~s}, 1 \mathrm{H}, \mathrm{N}=\mathrm{CH}) ;{ }^{13} \mathrm{C}$ NMR $\left(100 \mathrm{MHz}, \mathrm{DMSO}-\mathrm{d}_{6}\right): \delta 23.51\left(\mathrm{COC}_{3}\right), 56.29\left(\mathrm{OCH}_{3}\right),[112.91,115.59,117.78,120.94,126.67$, 
$127.48,138.72,142.10,149.10,141.25]$ (arom-C), [127.60, 127.98 (2C), 128.65 (2C), 130.09] ( $\mathrm{C}_{3}$ 'e bağl1 arom-C), 145.90 (triazol$\left.\mathrm{C}_{3}\right), 148.07(\mathrm{~N}=\mathrm{CH}), 151.51($ triazol-C 5$), 155.36(\mathrm{COO}), 166.30\left(\mathrm{COCH}_{3}\right) ; \mathrm{UV}$ [Etanol, $\left.\lambda_{\max }, \mathrm{nm}\left(\varepsilon, \mathrm{L}_{\mathrm{mol}}{ }^{-1} . \mathrm{cm}^{-1}\right)\right]: 256(20.800), 216$ (15.190).

\subsection{Yeni bileşiklerin antioksidan özelliklerinin incelenmesi}

\subsubsection{Indirgeme gücü tayini}

İndirgeme gücü tayini Oyaizu metodu kullanılarak yapılmıștır (Oyaizu, 1986). Farklı konsantrasyondaki örneklerin (50-250 $\mu \mathrm{g} / \mathrm{mL}$ ) dimetilsülfositte $(1 \mathrm{~mL})$ hazırlanan çözeltileri, fosfat tamponu $(2.5 \mathrm{~mL} ; 0.2 \mathrm{M} ; \mathrm{pH}=6.6)$ ve potasyum ferrisiyanür $(2.5 \mathrm{~mL} ; 1 \%)$ ile karıştııılmıştır. Deney tüpleri iyice karıştırılmış ve $50^{\circ} \mathrm{C}$ 'de 20 dakika inkübasyona bırakılmıştır. İnkübasyonun ardından oda sıcaklığına getirilen her bir deney tüpüne \% 10 'luk TCA çözeltisinden $2,5 \mathrm{~mL}$ ilave edilmiş ve 3000 rpm'de 10 dakika santrifüjlenmiştir. Süzüntüden $2,5 \mathrm{~mL}$ alınarak deney tüplerine aktarılmış ve çözeltiye $2,5 \mathrm{~mL}$ su, ardından $0,5 \mathrm{~mL} \mathrm{FeCl} l_{3}$ çözeltisinden eklenmiştir. Elde edilen renkli komplekslerin, UV spektrofotometresi ile $700 \mathrm{~nm}$ 'de ölçümü yapılmışıtır. Reaksiyon karışımının absorbansında meydana gelen artış ile numunenin indirgeme gücü arasında doğru bir orantı vardır.

\subsubsection{Serbest radikal giderme aktivitesi tayini}

Yeni sentezi gerçekleştirilen bileşiklerin serbest radikal giderme aktivitelerinin tayini DPPH'’nn kullanıldığı Blois metoduna göre yapılmıştır (Blois, 1998). Özetle, DPPH'’n etanoldeki $0.1 \mathrm{mM}$ çözeltisinden (1 mL), DMSO (3 mL) içinde farklı konsantrasyonlarda (50-250 $\mu \mathrm{g} / \mathrm{mL})$ örnek çözeltilere eklenmiştir. Deney tüpleri iyice karıştırılmış ve sonra oda sıcaklığında 30 dakika bekletilerek 517 nm'de UV spektrofotometresinde absorbansları ölçülmüş̧ür. Reaksiyon karışımının absorbansında görülen düşüş, numunenin serbest radikal giderme aktivitesi ile doğru orantılıdır. Reaksiyon ortamındaki DPPH radikalini giderme aktivitesi için hesaplamalar aşağıdaki formüle göre hesaplanmıştır:

$\%$ Serbest Radikal Giderme Aktivitesi $=\left(A_{0}-A_{1} / A_{0}\right) \times 100 ; A_{0}$ kontrol reaksiyonunun absorbans değeri iken, $A_{1}$ numune veya standardın absorbansıdır.

\subsubsection{Metal şelatlama aktivitesi tayini}

Metal şelat aktivitesi tayini Dinis metodu kullanılarak yapılmıştır (Dinis et al., 1994). Kısaca, deney tüplerine konulan numuneler ve standartlar 15, 30 ve $45 \mu \mathrm{g} / \mathrm{mL}$ konsantrasyonlarını oluşturacak ve toplam hacimleri $200 \mu \mathrm{L}$ olacak şekilde saf su kullanılarak seyreltilmiştir. Sonra sırasıyla $\mathrm{FeCl}_{2} \cdot 4 \mathrm{H}_{2} \mathrm{O}$, etil alkol ve ferrozin ilave edilerek karıştııılmış ve oda sıcaklığında 10 dakika bekletilmiştir. Oluşan renk 562 nm'de UV spektrofotometresinde köre (ferrozin dışındakiler) karşı okunmuştur. Reaksiyon karışımının absorbansındaki düşüş, numunenin metal şelatlama aktivitesi ile doğru orantılıdır. Ferrozin- $\mathrm{Fe}^{+2}$ kompleks oluşumunun inhibisyon yüzdesi aşağıdaki formülü kullanılarak hesaplanmıştır:

Şelat Yüzdesi $=\left(A_{0}-A_{1} / A_{0}\right) \times 100, A_{0}$ kontrol reaksiyonunun absorbans değeri iken, $A_{1}$ numune veya standardın absorbansıdır.

\section{Araştırma Sonuçları ve Tartışma}

Mevcut çalışmada, 3-alkil(aril)-4-amino-4,5-dihidro-1 H-1,2,4-triazol-5-on'ların (1), 2-formil-6-metoksifenil furan-2-karboksilat (2) ile reaksiyonundan 2-metoksi-6-[(3-alkil/aril-4,5-dihidro-1H-1,2,4-triazol-5-on-4-il)-azometin]fenil furan-2-karboksilatlar (3) sentezlenmiştir. Daha sonra, 3 bileşiğinin asetilasyon reaksiyonu ile 2-metoksi-6-[(1-asetil-3-alkil/aril-4,5-dihidro-1H-1,2,4-triazol-5on-4-il)-azometin]fenil furan-2-karboksilatlar (4) elde edilmiştir. 2-4 Bileşiklerinin yapıları IR, ${ }^{1} \mathrm{H}-\mathrm{NMR},{ }^{13} \mathrm{C}-\mathrm{NMR}$ ve UV spektral verileri kullanılarak aydınlatılmıştır.

\subsection{Antioksidan aktivite incelemeleri}

Sentezlenen on dört yeni bileşiğin, $\mathbf{3}$ ve $\mathbf{4}$, antioksidan aktivite incelemeleri yapılmıştır ve sonuçlar aşağıda verilmiş̧ir:

\subsubsection{Indirgeme gücü}

Sentezlenen bileşiklerin $700 \mathrm{~nm}$ 'de yapılan indirgeme gücü testleri sonucunda elde edilen bulgular, sentezlenen bileşiklerin absorbans değerlerinin, standartlara kıyasla çok daha düşük olması nedeniyle bileşiklerin indirgeyici özelliğe sahip olamadıklarını göstermektedir.

\subsubsection{Serbest radikal giderme aktivitesi}

Sentezlenen yeni bileşiklerin (3a-g, $\mathbf{4 a - g}$ ) ve kullanılan referans antioksidan maddelerin (BHT, BHA ve $\alpha$-tokoferol), radikal giderme aktivite testleri neticesinde elde edilen veriler aşağıdaki grafiğe (Şekil 1) geçirilmiştir. Grafikte sentezi gerçekleştirilen yeni bileşiklerin $517 \mathrm{~nm}$ 'de farklı derişimlerde yapılan ölçümlerinde, serbest radikal giderme aktiviteleri \% inhibisyon olarak verilmektedir. Elde edilen veriler doğrultusunda; yeni sentezlenen $3 \mathbf{e}, \mathbf{3 f}$ ve $\mathbf{4 c - 4 g}$ bileşiklerinin konsantrasyona bağlı olarak aktivitelerinde artış olduğu halde, diğer bileşiklerde böyle bir durumun olmadığı anlaşılmıştır. Sonuç olarak yine de bahsi geçen bileşikler etkin radikal giderici özelliğe sahip değildir. 


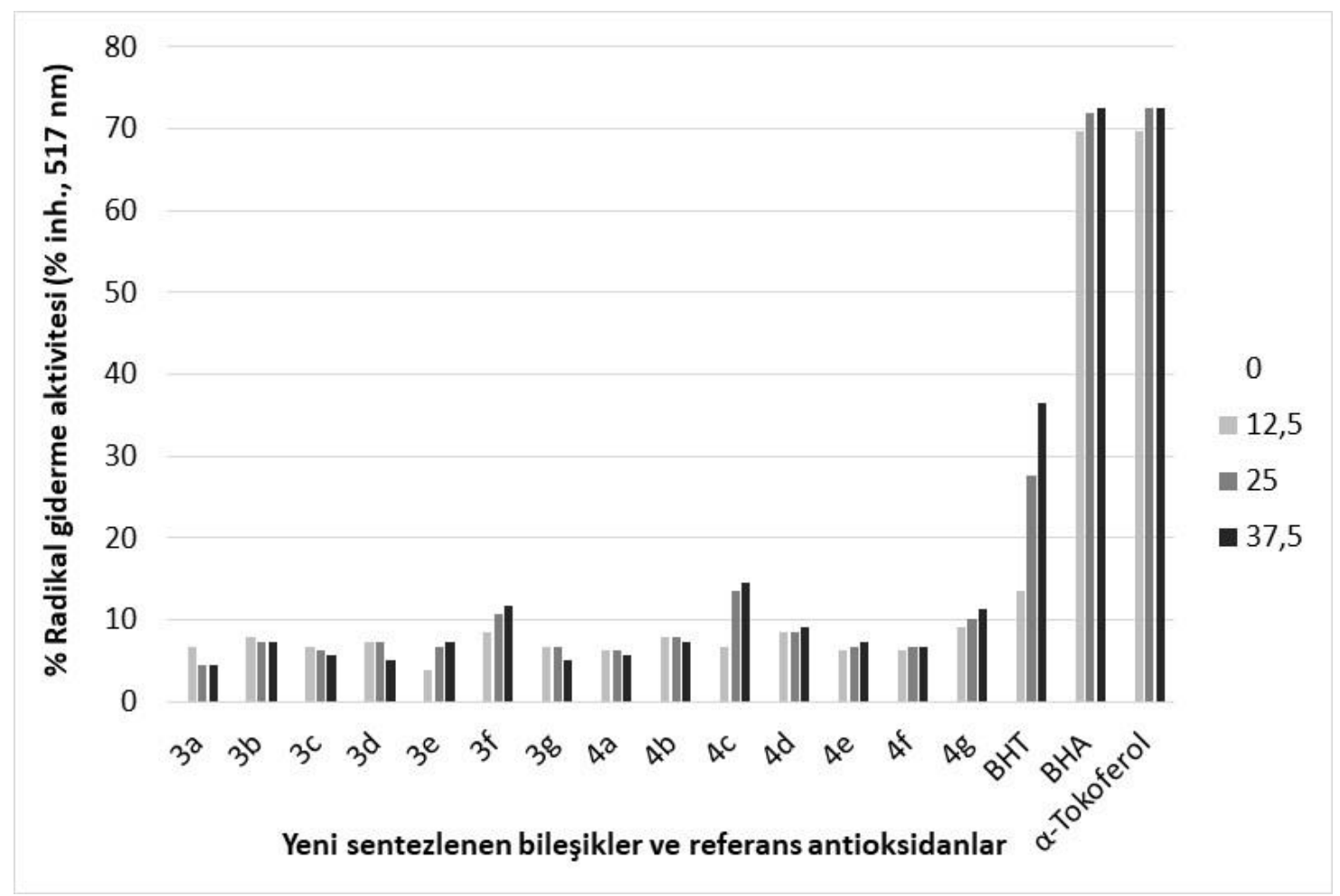

Şekil 1. 3, 4 Bileşiklerinin ve referans antioksidanların farklı konsantrasyonlardaki radikal giderme aktiviteleri

\subsubsection{Metal şelat aktivitesi}

Genellikle, yapısında - $\mathrm{OH},-\mathrm{SH},-\mathrm{COOH},-\mathrm{PO}_{3} \mathrm{H}_{2}, \mathrm{C}=\mathrm{O},-\mathrm{NR}_{2},-\mathrm{S}-$, -O- gibi iki veya daha fazla fonksiyonel grubu uygun bir yapıişlev konfigürasyonu ile içeren bileşiklerin şelasyon aktivitesi göstereceği bildirilmiştir (Hussain ve ark., 2003; McClements ve Decker, 2000). (Lindsay, 1996; Yuan ve ark., 2005; Gülçin, 2006). Bu açıdan bakıldığında, L-karnitin için demir iyonlarını hidroksil ve karboksilat gruplarıyla şelatladığı düşünülmektedir (Gülçin, 2006). Bu çalışmada; 3 ve 4 tipi bileşikler -O- ve $\mathrm{C}=\mathrm{O}$ gruplarını içermektedir. Hatta 3 tipi bileşiklerin asetilasyon reaksiyonu ile daha etkili şelatörlere dönüştürülmeleri planlanmıştır. Bu amaçla, $\mathbf{3}$ ve 4 bileşikleri ile EDTA ve $\alpha$-tokoferol referans antioksidanlarının metal şelatlama aktiviteleri incelenmiştir. Şekil 2'deki grafikte aktiviteleri \% inhibisyon olarak gösterilmektedir. Tüm beklentilere karşın yeni bileşiklerin düşük konsantrasyonda düşük aktiviteye sahip oldukları görülmektedir, yani etkin şelatörler değillerdir. 


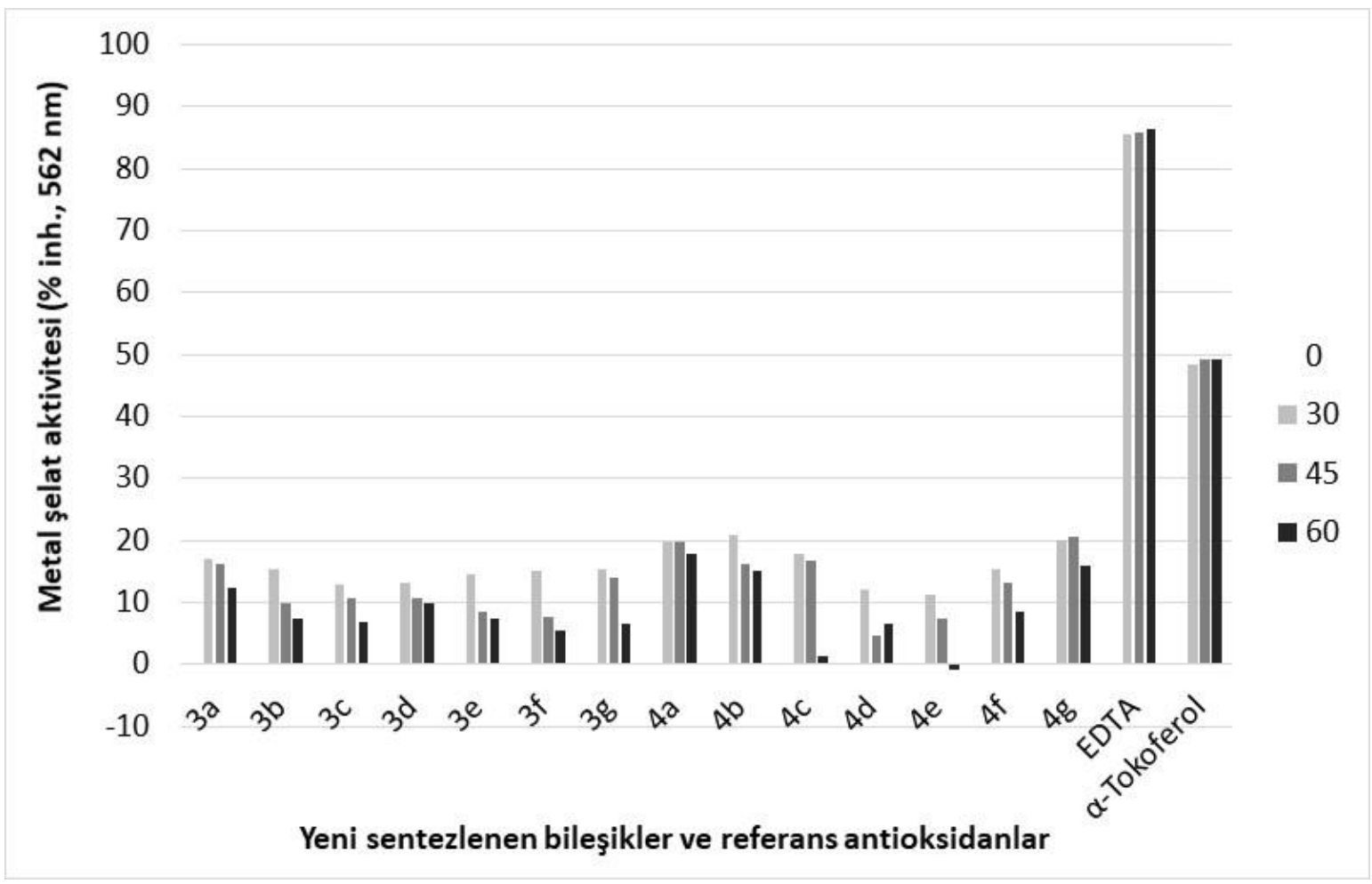

Şekil 2. 3, 4 Bileşiklerinin ve referans antioksidanların farkl konsantrasyonlardaki metal şelatlama aktiviteleri

\section{Sonuç}

$\mathrm{Bu}$ çalışmada, umut verici bir biyolojik ajan olan 1,2,4-triazol halkasını yapısında içeren yeni 2-metoksi-6-[(3-alkil/aril-4,5-dihidro1H-1,2,4-triazol-5-on-4-il)-azometin]fenil furan-2-karboksilatlar ve asetil türevleri sentezlenmiştir. Sentezlenen bileşiklerin yapıları IR, ${ }^{1} \mathrm{H}$ NMR, ${ }^{13} \mathrm{C}$ NMR ve UV spektral verileri kullanılarak karakterize edilmiştir. Son olarak sentezlenen bileşiklerin üç farklı yöntemle in vitro antioksidan özellikleri incelenmiştir. Bileşiklerin antioksidan aktivite sonuçlarına göre umut verici bir aktivite göstermedikleri belirlenmiştir.

\section{Teșekkür}

Bu çalışma (107T633) no’lu proje kapsamında TÜBİTAK tarafından desteklenmiştir.

\section{Kaynakça}

Aktas-Yokus, Ö., Yuksek, H., Manap, S., Aytemiz, F., Alkan, M., Beytur, M., Gürsoy-Kol, Ö. (2017). In-vitro biological activity of some new 1,2,4-triazole derivatives with their potentiometric titrations. Bulg. Chem. Commun., 49: 98-106.

Ali, K.A., Ragab, E.A., Farghaly, TA, Abdalla, M.M. (2011). Synthesis of new functionalized 3-substituted [1,2,4]triazolo [4,3-a] pyrimidine derivatives: Potential antihypertensive agents. Acta Pol. Pharm., 68(2): 237-247.

Alkan, M., Yüksek, H., Gürsoy-Kol, Ö., Calapoğlu, M. (2008). Synthesis, acidic and antioxidant properties of some novel 3,4disubstituted-4,5-dihydro-1H-1,2,4-triazol-5-one derivatives, Molecules, 13: 107-121.

Bahçeci, S., Yüksek, H., Ocak, Z., Köksal, C., Özdemir, M., (2002). Synthesis and non-aqueous medium titrations of some new 4,5dihydro-1H-1,2,4-triazol-5-one derivative, Acta Chem. Slov., 49: 783-794.

Blois, M. (1958). Antioxidant determinations by the use of a stable free radical. Nature, 181(4617): 1199-1200.

Chidananda, N., Poojary, B., Sumangala, V., Kumari, N.S., Shetty, P., Arulmoli, T. (2012). Facile synthesis, characterization and pharmacological activities of 3,6-disubstituted 1,2,4-triazolo[3,4-b][1,3,4]thiadiazoles and 5,6-dihydro-3,6-disubstituted-1,2,4triazolo[3,4-b][1,3,4]thiadiazoles. Eur. J. Med. Chem. 51: 124-136.

Demirbas, N., Ugurluoglu, R., Demirbas. A. (2002). Synthesis of 3-alkyl(Aryl)-4-alkylidenamino-4,5-dihydro-1H-1,2,4-triazol-5-ones and 3-alkyl-4-alkylamino-4,5-dihydro-1H-1,2,4-triazol-5-ones as antitumor agents. Bioorg. Med. Chem., 10(12): 3717-3723.

Dinis, T.C.P., Madeira, V.M.C., Almeida, L.M. (1994). Action of phenolic derivatives (Acetaminophen, salicylate, and 5aminosalicylate) as inhibitors of membrane lipid peroxidation and as peroxyl radical scavengers. Arch. Biochem. Biophys, 315(1): 161-169. 
Gülçin, I. (2006). Antioxidant and antiradical activities of L-carnitine. Life Sci., 78: 803-811.

Gürsoy-Kol, Ö., Ayazoglu, E. (2017). Antioxidant activities and acidic properties of some novel 4-[3,4-di-(4-nitrobenzoxy)benzylidenamino]-4,5-dihydro-1H-1,2,4-triazol-5-one derivatives. Arab. J. Chem., 10(S2): S2881-S2889.

Gürsoy Kol, Ö. Yüksek, H. (2010). Synthesis and in-vitro antioxidant evaluation of some novel 4,5-dihydro-1H-1,2,4-triazol-5-one derivatives, E-J. Chem., 7(1): 123-136.

Henen, M.A., El Bialy, S.A.A., Goda, F.E., Nasr, M.N.A., Eisa, H.M. (2012). [1,2,4]Triazolo[4,3-a]quinoxaline: Synthesis, antiviral, and antimicrobial activities. Med. Chem. Res., 21(9): 2368-2378.

Hussain, H.H, Babic, G., Durst, T, Wright, J.S., Flueraru, M., Chichirau, A., Chepelev, L.L. (2003). Development of novel antioxidants: Design, synthesis, and reactivity. J. Org. Chem., 68(18): 7023-7032.

Ikizler, A., Yüksek, H. (1993). Acetylation of 4-amino-4,5-dihydro-1H-1,2,4-triazol-5-ones. Org. Prep. Proc. Int., 25(1): 99-105.

Ikizler, A.A., Johansson, C.B., Bekircan, O., Çelik, C. (1999). Synthesis and antibacterial activities of some 1,2,4-triazole derivatives. Acta Pol. Pharm. - Drug Res., 56(4): 283-288.

Li, Z., Cao, Y., Zhan, P., Pannecouque, C., Balzarini, J., Clercq, E.De. (2013). Synthesis and anti-HIV evaluation of novel 1,2,4-triazole derivatives as potential non-nucleoside HIV-1 reverse transcriptase inhibitors. Lett. Drug Des. Discov., 10(1): 27-34.

Lindsay, R.C. (1996). Food additives. In: Fennema, O.R. (Ed.), Food Chemistry. Marcel Dekker Inc., New York, pp. 778-780 (Chapter 12).

McClements, D., Decker, E. (2000). Lipid oxidation in oil-in-water emulsions: Impact of molecular environment on chemical reactions in heterogeneous food systems. J. Food Sci., 65(8): 1270-1282.

Oyaizu, M. (1986). Studies on products of browning reaction. Antioxidative activities of products of browning reaction prepared from glucosamine. Japanese J. Nutr. Diet., 44(17): 307-315.

Saadeh, H.A., Mosleh, I.M., Al-Bakri, A.G., Mubarak, M.S. (2010). Synthesis and antimicrobial activity of new 1,2,4-triazole-3-thiol metronidazole derivatives. Monats. Chem., 141(4): 471-478.

Uzgören-Baran, A., Tel, B.C., Sarıöl, D., Öztürk, E.I., Kazkayası, I., Okay, G., Ertan, M., Tozkoparan, B. (2012). Thiazolo[3,2-b]1,2,4-triazole-5(6H)-one substituted with ibuprofen: Novel non-steroidal anti-inflammatory agents with favorable gastrointestinal tolerance. Eur. J. Med. Chem., 57: 398-406.

Yuan, Y.V., Bone, D.E., Carrington, M.F. (2005). Antioxidant activity of dulse (Palmaria palmata) extract evaluated in vitro. Food Chem., 91: 485-494.

Yüksek, H., Akyıldırım, O., Yola, M.L., Gürsoy-Kol, Ö., Çelebier, M., Kart, D. (2013). Synthesis, in vitro antimicrobial and antioxidant activities of some new 4,5-dihydro-1H-1,2,4-triazol-5-one derivative, Arch. Pharm., 346(6): 470-480.

Yüksek, H., Üçüncü, O., Alkan, M., Ocak, Z., Bahçeci, Ş. (2005). Non-aqueous medium titrations of some new 4-benzylidenamino4,5-dihydro-1H-1,2,4-triazol-5-one derivatives", Molecules, 10: 961-970. 